\title{
Cefepime challenge after piperacillin/tazobactam-induced thrombocytopenia
}

\author{
Caroline Beaulieu $^{1} \cdot$ Lisa Kurczewski $^{1} \cdot$ Vishal Yajnik $^{2}$
}

Published online: 9 April 2019

○) Springer Science+Business Media, LLC, part of Springer Nature 2019

\begin{abstract}
Drug-induced thrombocytopenia (DITP) has been described as a sudden and severe hematologic complication of piperacillin/tazobactam. The proposed mechanism by which piperacillin/tazobactam causes DITP involves the formation of a covalent bond to platelet membrane protein thereby inducing a humoral immune response. Given the immunogenic nature of this adverse event and the structural similarities across beta-lactam antibiotics, the potential for cross-reactivity between agents within the class should be considered. However, the structural moiety of piperacillin/tazobactam responsible for this immunogenic response has not been identified - the relationship between structure and activity for this phenomenon remains unknown. Data on the safety and cross-reactivity of other beta-lactam agents in this setting is lacking. We report the first case of piperacillin/tazobactam DITP successfully challenged by the use of cefepime for the treatment of aspiration pneumonia. Further studies are needed to determine the structural moiety of piperacillin/tazobactam responsible for this immunogenic response and evaluate the safety of other beta-lactam antibiotics in this clinical setting.
\end{abstract}

Keywords Beta-lactams $\cdot$ Cefepime $\cdot$ Cross reactions $\cdot$ Piperacillin $\cdot$ Thrombocytopenia

\section{Highlights}

- Piperacillin/tazobactam drug-induced thrombocytopenia (DITP) is immunogenic in nature.

- Data on the safety and cross-reactivity of other betalactam agents in this setting is lacking.

- We report a case of piperacillin/tazobactam DITP successfully challenged with cefepime.

- Studies are needed to assess the use of other beta-lactam agents and structural moiety involved.

Caroline Beaulieu

dr.carolinebeaulieu@gmail.com

1 Department of Pharmacy, Virginia Commonwealth University Health, 401 North 12th Street, P.O. Box 980042 , Richmond, VA 23298-0042, USA

2 Department of Anesthesiology, Virginia Commonwealth University Health/Medical College of Virginia Hospitals, 1200 E. Broad Street, 7th Floor, P.O. Box 980695, Richmond, VA 23298-0695, USA

\section{Introduction}

Thrombocytopenia is a serious hematological finding with a reported incidence ranging from $15-58 \%$ in intensive care unit (ICU) patients [1]. A platelet count nadir below $100,000 / \mu \mathrm{L}$ and a decrease in platelet count of $\geq 30 \%$ has been associated with higher mortality in this patient population [2]. Although this condition is commonly encountered in clinical practice, diagnosis remains a challenge. The etiology of thrombocytopenia is often complex and multifactorial $[3,4]$.

Several medications can be implicated in the development of thrombocytopenia [5]. As such, drug-induced thrombocytopenia (DIT) should always be considered in the initial evaluation. DIT is further classified based on the mechanism of thrombocytopenia. Medications can either decrease platelet production secondary to myelosuppression or accelerate platelet destruction via immune-mediated processes [6]. Although beta-lactam antibiotics may cause bone marrow suppression, drug-induced immune-mediated thrombocytopenia (DITP) is more common with this class [7-9]. In contrast to myelosuppression, DITP often presents earlier. In treatment-naïve patients, profound thrombocytopenia can be observed within 7-14 days [10]. However, a decline in 
platelet count to below $5,000 / \mu \mathrm{L}$ as early as 2.5 hours postdose has been described in a patient with prior exposure [7].

Two mechanisms underlying piperacillin/tazobactaminduced immune-mediated thrombocytopenia have been proposed [5]. Piperacillin may bind covalently to platelet membrane protein and form immunogenic drug-protein adducts thereby inducing antibody production [5]. The drug may also stimulate the production of pre-existing antibodies that develop a higher affinity to platelet membrane protein only in the presence of the soluble drug [5, 10-13]. Due to the immunogenic nature of this reaction and the structural similarities between beta-lactam antibiotics, cross-reactivity may exist between certain agents within the class. However, the safety of other beta-lactam antibiotics in patients with piperacillin/tazobactam DITP remains uncertain. We report a case of piperacillin/tazobactam-mediated immune thrombocytopenia, subsequently treated successfully with cefepime.

\section{Case presentation}

A 40-year-old woman presented with a pontine hemorrhage in the setting of malignant hypertension. Her medical history was significant for type 1 diabetes, and systemic lupus erythematosus (untreated). She had also sustained a recent tibial fracture, for which she was started on thromboembolism prophylaxis with enoxaparin following operative fixation. Upon arrival to the emergency department, she was noted to have altered mental status with active vomiting and required intubation for airway protection. On ICU day 2, she was started on broad-spectrum antibiotic coverage with vancomycin and piperacillin/tazobactam for suspected aspiration pneumonia. Her baseline complete blood count (CBC) revealed a hemoglobin of $11.5 \mathrm{~g} / \mathrm{dL}$, a white blood cell count of $13,700 / \mu \mathrm{L}$ and a platelet count of $295,000 / \mu \mathrm{L}$. Owing to a low suspicion of methicillin-resistant Staphylococcus aureus infection, vancomycin was discontinued on ICU day 5 and a 7-day treatment course was completed with piperacillin/ tazobactam.

On ICU day 9, the patient was liberated from mechanical ventilation but subsequently aspirated and required reintubation two days later. In light of her acute pulmonary deterioration, fever, rising leukocytosis, and computed tomography (CT) of the chest consistent with pneumonia, vancomycin and piperacillin/tazobactam were resumed. The platelet count was $455,000 / \mu \mathrm{L}$ prior to the first dose of antibiotics. Her medication regimen had otherwise remained unchanged. On ICU day 11, a CBC obtained two hours after re-exposure to piperacillin/tazobactam revealed a platelet nadir of $<2,000 / \mu \mathrm{L}$ and a hemoglobin of $6.7 \mathrm{~g} / \mathrm{dL}$. She was noted to have an increase in bleeding from her oropharynx and ventilator circuit. Furthermore, a repeat head CT showed an interval increase in pontine hemorrhage. The patient subsequently received one additional dose of piperacillin/tazobactam before the medication was discontinued.

Given the timing and rate of decrease in platelet count, a diagnosis of piperacillin/tazobactam DITP was made. After hematology consultation, she was transfused two units of packed red blood cells on ICU day 12. Treatment with dexamethasone and intravenous immunoglobulin (IVIG) was started and her antibiotic regimen was changed to cefepime and metronidazole. She was placed back on subcutaneous heparin and acetaminophen. Her platelet count remained undetectable until 45 hours after the last dose of piperacillin/tazobactam and continued to recover thereafter (Fig. 1). She was subsequently treated for aspiration pneumonia with a 7-day course of cefepime without complications.

On ICU day 17, blood samples were sent to the Blood Center of Wisconsin for the detection of drug-dependent platelet-reactive antibodies to piperacillin/tazobactam using immunofluorescence by flow cytometry. However, per the platelet and neutrophil immunology laboratory at the Blood Center of Wisconsin, the 6-day delay between the last dose of piperacillin/tazobactam and blood sample collection resulted in failure to detect the presence of piperacillindependent platelet-reactive antibodies. Other factors that may have reduced the likelihood of detection included the administration of immunoglobulin and corticosteroids. As such, these results could not be used to rule out a diagnosis of DITP.

\section{Discussion}

Thrombocytopenia is a common hematological finding in critically ill patients $[1,14]$, with several pre-existing conditions and exogenous factors as potential causes [3, 4]. The effect of beta-lactam antibiotics on blood components has been described in the literature [13], with thrombocytopenia noted as one of their potential adverse sequelae [13].

DITP has been described as a sudden and severe hematologic complication of piperacillin/tazobactam [15-17]. The difficulty in determining which moiety of the medication's chemical structure is responsible for the immune response further complicates our ability to ascertain the risk of crossreactivity between other beta-lactam antibiotics.

To date, four reports have described the use of carbapenems in patients with piperacillin/tazobactam DITP $[8,9,11$, 17]. Only one case reports the substitution of piperacillin/ tazobactam with a different cephalosporin, cefoperazone, in this clinical setting [11]. The case of our patient is unique as it describes the use of cefepime for the treatment of aspiration pneumonia in a patient with piperacillin/tazobactam DITP. 


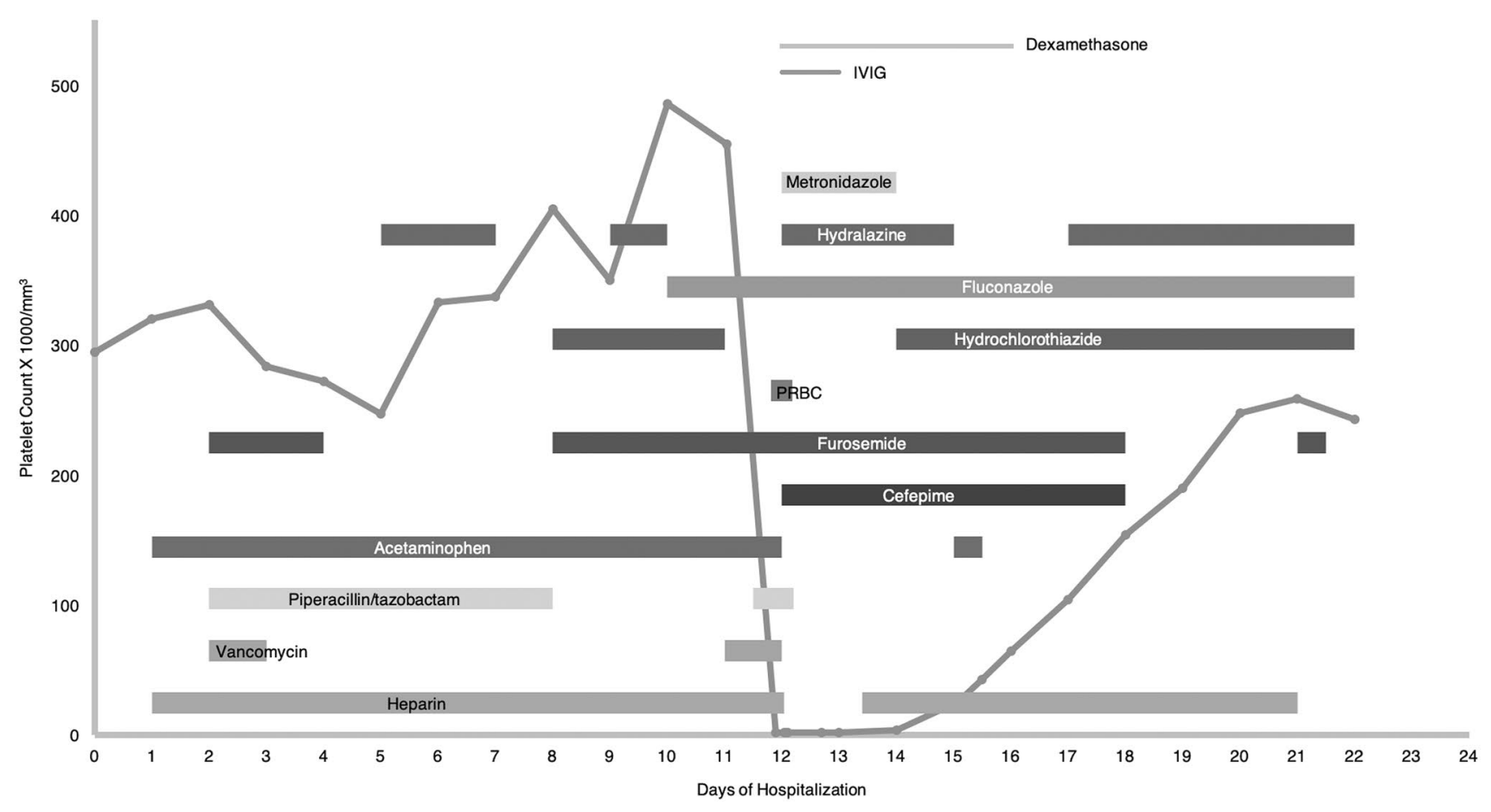

Fig. 1 Depiction of the patient's time course of thrombocytopenia and exposure to potential medication causes

The rate of platelet decline, absolute platelet count, and timing between critical platelet count drop and administration of piperacillin/tazobactam in the present case fits most closely with immune-mediated platelet destruction, as seen in DITP [5]. A platelet nadir of $<2,000 / \mu \mathrm{L}$ was observed on ICU day 11, two hours after piperacillin/tazobactam was reintroduced, and after the patient had completed a 7-day treatment course (ICU day 2-8). The platelet count recovered after the medication was discontinued.

Diagnosis of DITP secondary to piperacillin/tazobactam was further supported by the Naranjo Adverse Event Probability Scale [18]. Points were given for the existence of previous conclusive reports on this reaction $(+1)$, the reaction improving when the medication was discontinued $(+1)$ and the adverse event occurring after the suspected drug was administered (+2). The total score of four points categorizes this clinical outcome as a 'possible' adverse reaction to piperacillin/tazobactam.

Since thrombocytopenia is often multifactorial, other potential etiologies were investigated. Given the patient's past medical history of lupus erythematous, we examined immune thrombocytopenia purpura (ITP), splenomegaly, and platelet consumption in the setting of a thrombotic microangiopathic process as potential causes. As the patient's thrombocytopenia coincided with anemia and her platelet count varied considerably in the presence of concurrent illnesses and treatments, clinical suspicion for ITP was low. The absence of schistocytes on blood smear and a normal total bilirubin $(0.5 \mathrm{mg} / \mathrm{dL})$ led to the exclusion of microangiopathic processes. Although critical illness is a significant risk factor for thrombocytopenia, coagulation factors on ICU day 12 did not fit the classic diagnostic profile of disseminated intravascular coagulation (prothrombin time $=14.5 \mathrm{~s}$, activated partial thromboplastin time $=33 \mathrm{~s}$, fibrinogen $=594 \mathrm{mg} / \mathrm{dL}$ ). Lastly, splenic sequestration was deemed unlikely as CT imaging of the abdomen/pelvis did not reveal splenomegaly.

Other concurrent medications implicated as potential triggers of DIT were also examined. Although heparin can be associated with low platelet count, a low pretest clinical probability score (4T score) of one to two, a negative antiplatelet 4/heparin enzyme-linked immunosorbent assay (Optical density $=0.494$ ) and a negative serotonin assay ruled out heparin-induced thrombocytopenia (HIT) [14]. Furthermore, subcutaneous heparin was later resumed without complications and the degree of platelet drop did not fit the typical presentation of heparin-induced thrombocytopenia. Other medications that have previously been described as potential causes of thrombocytopenia and administered to our patient include vancomycin, metronidazole, acetaminophen, hydrochlorothiazide, fluconazole, furosemide, and hydralazine (Fig. 1) [19]. However, administration of those medications (except vancomycin) was resumed shortly after the platelet count nadir occurred and continued as the platelet count simultaneously recovered (Fig. 1). Although vancomycin was discontinued on ICU day 12 , a sub-therapeutic 
level remained during platelet recovery, as evidenced by a random vancomycin level of $5.1 \mathrm{mg} / \mathrm{L}$ on ICU day 16 . As vancomycin-induced thrombocytopenia has previously been reported at such levels, vancomycin DITP was also ruled out [20].

In conclusion, we report the first case of piperacillin/tazobactam DITP successfully challenged by the use of cefepime for the treatment of aspiration pneumonia. In light of the immunogenic nature of this adverse event and the structural similarities between beta-lactam antibiotics, the potential for cross-reactivity between agents within that class should be considered. Further studies are needed to determine the structural moiety of piperacillin/tazobactam responsible for this immunogenic response and evaluate the safety of other beta-lactam antibiotics in this clinical setting. Particular emphasis should be placed on the role of chemical structure in determining the risk of DITP between beta-lactam agents.

Author contribution V. Yajnik, L. Kurczewski, and C. Beaulieu are the primary contributors to the article and idea development. V. Yajnik, L. Kurczewski, and C. Beaulieu are the primary providers of the patient cases and contributed to the editorial revisions of the article.

Funding This research did not receive any specific grant from funding agencies in the public, commercial, or not-for-profit sectors.

\section{Compliance with ethical standards}

Conflict of interest V. Yajnik, L. Kurczewski and C. Beaulieu declare that they have no conflict of interest.

Ethical statement The patient is deceased and did not have mental or physical capacity to provide signed consent at any point during their care due to their medical condition. Given the circumstances, we took additional steps to protect the patient's privacy and anonymize the manuscript to the greatest possible extent. Furthermore, all information has been de-identified and unique characteristics have been excluded.

\section{References}

1. Moreau D, Timsit JF, Vesin A, Garrouste-Orgeas M, de Lassence A, Zahar JR et al (2007) Platelet count decline, an early prognostic marker in critically ill patients with prolonged ICU stays. Chest 131:1735-1740

2. Strauss R, Wehler M, Mehler K, Kreutzer D, Koebnick C, Hahn EG (2002) Thrombocytopenia in patients in the medical intensive care unit: bleeding prevalence, transfusion requirements, and outcome. Crit Care Med 30:1765-1771
3. McMillan R (2007) Hemorrhagic disorders: abnormalities of platelet and vascular function. In: Goldman L, Ausiello D (eds) Cecil medicine, 23rd edn. Saunders Elsevier, Philadelphia, pp $1289-1300$

4. Janz TG, Hamilton GC (2006) Disorders of hemostasis. In: Marx J (ed) Rosen's emergency medicine: concepts and clinical practice, 7th edn. Mosby Elsevier, Philadelphia, pp 1578-1589

5. Aster RH, Bougie DW (2007) Drug-induced immune thrombocytopenia. N Engl J Med 357:580-587

6. Visentin GP, Liu CY (2007) Drug-induced thrombocytopenia. Hematol Oncol Clin North Am 21:685-696

7. Boyce K, Brar H, Stabler SN (2016) Piperacillin/tazobactaminduced immune-mediated thrombocytopenia in the intensive care unit. J Clin Pharm Ther 41:730-732

8. Nguyen VD, Tourigny JF, Roy R, Brouillette D (2015) Rapidonset thrombocytopenia following piperacillin-tazobactam reexposure. Pharmacotherapy 35:326-330

9. Shaik S, Kazi HA, Ender PT (2015) Rapid-onset piperacillintazobactam induced thrombocytopenia. J Pharm Pract 28:204-206

10. Loo AS, Gerzenshtein L, Ison MG (2012) Antimicrobial druginduced thrombocytopenia: a review of the literature. Semin Thromb Hemost 38:818-829

11. Chen H, Fan Z, Guo F, Yang Y, Li J, Zhang J et al (2016) Tazobactam and piperacillin-induced thrombocytopenia: a case report. Exp Ther Med 11:1223-1226

12. Leger RM, Arndt PA, Garratty G (2008) Serological studies of piperacillin antibodies. Transfusion 48:2429-2434

13. Bang NU, Kammer RB (1983) Hematologic complications associated with beta-lactam antibiotics. Rev Infect Dis 5(2 Suppl):S380-S393

14. Greinacher A, Selleng K (2010) Thrombocytopenia in the intensive care unit patient. ASH Educ Progr 2010:135-143

15. Pérez-Vázquez A, Pastor JM, Riancho JA (1998) Immune thrombocytopenia caused by piperacillin/tazobactam. Clin Infect Dis 27:650-651

16. Yan MT, Chu HY, Chau T, Lin SH (2009) Profound thrombocytopenia associated with piperacillin in a hemodialysis patient. Clin Nephrol 72:240-243

17. Alzahrani M, Alrumaih I, Alhamad F, Abdel Warith A (2018) Rapid onset sudden thrombocytopenia following reexposure to piperacillin-tazobactam: report of two cases and review of the literature. Platelets 29:628-631

18. Naranjo CA, Busto U, Sellers EM, Sandor P, Ruiz I, Roberts EA et al (1981) A method for estimating the probability of adverse drug reactions. Clin Pharmacol Ther 30:239-245

19. Wazny LD, Ariano RE (2000) Evaluation and management of drug-induced thrombocytopenia in the acutely ill patient. Pharmacotherapy 20:292-307

20. Mohammadi M, Jahangard-Rafsanjani Z, Sarayani A, Hadjibabaei M, Taghizadeh-Ghehi M (2017) Vancomycin-induced thrombocytopenia: a narrative review. Drug Saf 40:49-59

Publisher's Note Springer Nature remains neutral with regard to jurisdictional claims in published maps and institutional affiliations. 